\title{
Laboratory Diagnosis of Leptospirosis
}

\author{
Ahmad SN, Shah S, Ahmad FMH*
}

Metropolis Health

Services (India) Pvt Ltd,

*Indian Army, Mumbai, India

Correspondence:

S Ahmad

Email:

nishaahmad@metropolisindia.com

\begin{abstract}
Leptospirosis is a worldwide zoonosis caused by pathogenic Leptospira species, for which humans are accidental hosts. It is endemic in the tropical urban areas including our country, where seasonal epidemics are becoming increasingly common. Laboratory tests are necessary to confirm the diagnosis of clinically suspected leptospirosis due to its varied symptomatology. Moreover, leptospirosis must always be considered during the differential diagnosis of other tropical febrile illnesses .Laboratory analysis depends on the samples available and temporal stage of the illness. A confusing array of laboratory tests is described for the detection of this spirochete and antibodies. The conventional tests include direct microscopy, culture and the most widely used reference standard method -the microscopic agglutination test. In addition a variety of newer serological tests and those based on molecular techniques have been described.

This review has attempted to describe the basis of these techniques and discussed the relative advantages and drawbacks of these assays with special emphasis on the selection of the most appropriate specimen and test, and the correct interpretation of the test result
\end{abstract}

PubMed ID : 16333192

J Postgrad Med 2005;51:195-200

\section{KEY WORDS: Leptospira, Investigations, Isolation}

eptospirosis is one of the most widespread of zoonoses, and of special importance in the Indian setting where it has emerged as an important cause of febrile illness with seasonal and geographical variation. ${ }^{[1]}$ This eminently treatable infection of ubiquitous distribution is caused by pathogenic spirochetes -Leptospira species, which have been classified into serovars based on their antigenic characteristics and recently into species based on genomic studies. ${ }^{[2]}$

Infected rodents and other wild and domestic animals pass the bacteria in their urine. The bacteria can survive for a long time in fresh water, damp soil, vegetation, and mud. Flooding after heavy rainfall helps spread the bacteria in the environment. Human leptospirosis is caused by contact through broken skin and mucous membranes with fresh water, damp soil, or vegetation contaminated by the urine of infected animals or ingestion.

Bacteremia spreads the spirochete throughout the body and causes signs and symptoms of illness. The spectrum of human disease is extremely wide, ranging from subclinical infection to a severe syndrome of potentially fatal, multiorgan dysfunction. Most infected persons have a mild to moderate illness that is similar to many other tropical diseases. Symptoms include fever, headache, chills, nausea,vomiting, eye inflammation and muscle aches. In more severe cases, the illness can result in liver damage and jaundice, kidney failure, and internal bleeding. ${ }^{[3]}$

The mechanisms by which leptospires cause disease are not well understood. A number of putative virulence factors have been suggested, but with few exceptions their role in pathogenesis remains as yet undefined.

A large array of tests has been described, but in practice, availability of appropriate laboratory support is still a problem ${ }^{[4,5]}$. Hence, laboratory diagnosis of leptospirosis is a confusing topic for personnel involved in leptospirosis diagnosis, treatment and surveillance.Conventionally either culture positivity, or, recent seroconversion, or a four fold rise in microagglutinin titres (MAT) should be demonstrated for confirmation. ${ }^{[6,7,8]}$

This review seeks to discuss the laboratory findings in Leptospirosis with emphasis on the utility of various diagnostic tests [Table 1].

\section{Non-specific laboratory findings}

The erythrocyte sedimentation rate (ESR) is elevated in anicteric disease, and white cell counts (WBC) range from below normal to moderately elevated. Liver function tests (LFT) show a slight elevation in aminotransferases, bilirubin, and alkaline phosphatase. Urinalysis shows proteinuria, pyuria, and often microscopic hematuria. Hyaline and granular casts may also be present during the first week of illness.

Lumbar puncture usually reveals a normal or slightly elevated CSF pressure. CSF examination may initially show a predominance of polymorphs or lymphocytes, but later examination almost invariably shows that lymphocytes predominate. CSF 
Table:1 Summary of laboratory diagnosis of leptospirosis

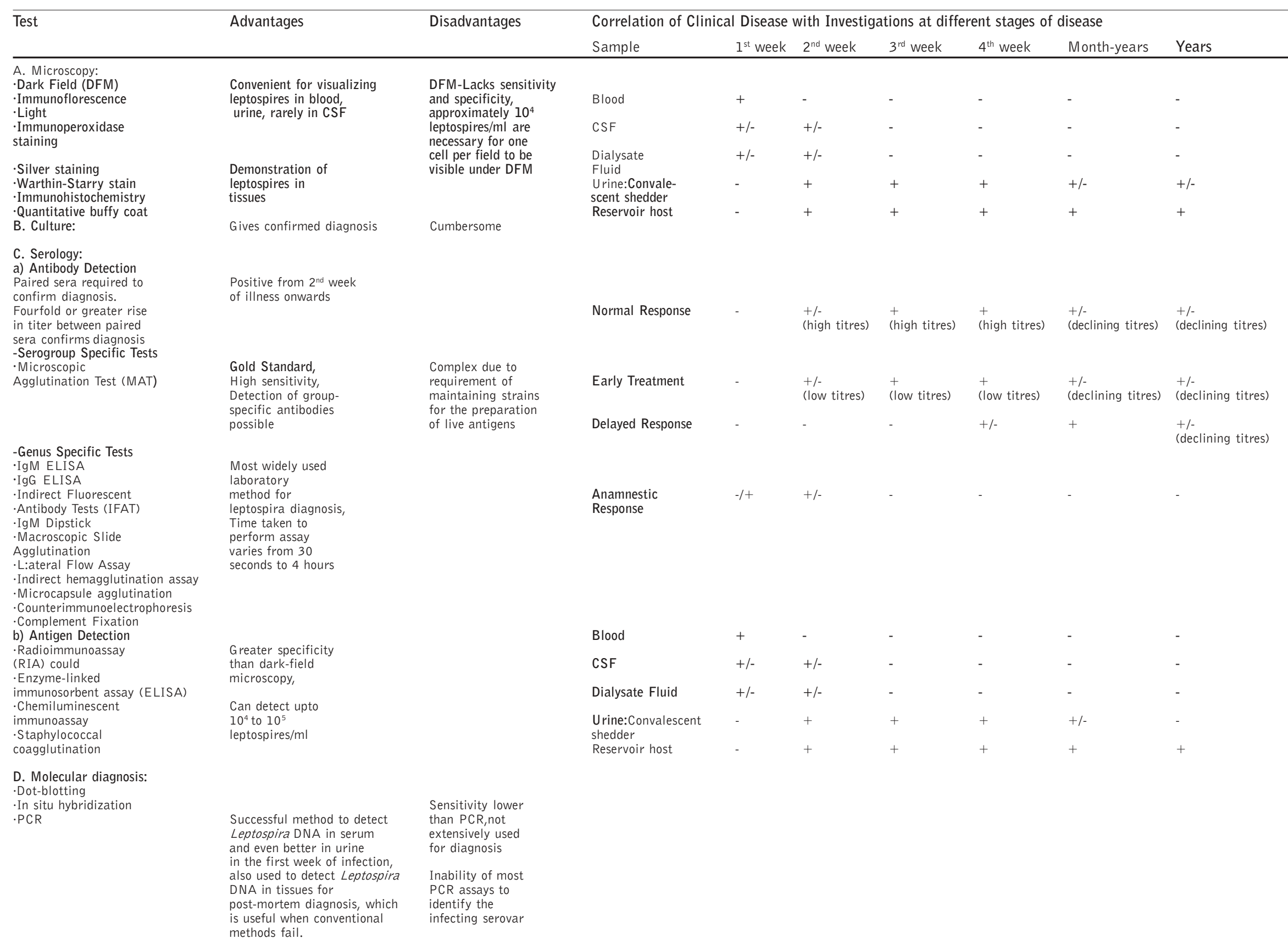


protein may be normal or elevated, while CSF glucose is usually normal. In patients with severe jaundice, xanthochromia may occur. CSF abnormalities are common in the second week of illness, and CSF pleocytosis can persist for weeks.

In severe leptospirosis, a peripheral leukocytosis with a shift to the left is not uncommon. Thrombocytopenia is common and may be marked. Renal function impairment is indicated by raised plasma creatinine levels. The degree of azotemia varies with severity of illness.

In icteric leptospirosis, liver function tests (LFT) generally show a significant rise in bilirubin, with lesser increase in transaminases and marginal increase in alkaline phosphatase levels. The hyperbilirubinemia is generally out of proportion to the other liver function test values. Serum amylase may also be elevated, particularly in patients with acute renal failure.

The nonspecific nature of these changes can only suggest a diagnosis of leptospirosis. For confirmation of the diagnosis, specific microbiological tests are necessary.

\section{Diagnostic tests}

\section{Microscopic Demonstration}

Leptospires may be visualized in clinical material by dark-field microscopy or by immunofluorescence or light microscopy after appropriate staining. Approximately $10^{4}$ leptospires $/ \mathrm{ml}$ are necessary for one cell per field to be visible by dark-ground microscopy (DGM). A quantitative buffy coat method has been shown to have a sensitivity of approximately $10^{3}$ leptospires/ $\mathrm{ml}$. Microscopy of blood is of value only during the first few days of the acute illness, while leptospiremia occurs. Dark-field microscopic examination of body fluids such as blood, urine, CSF, and dialysate fluid has been used but is both insensitive and lacks specificity. The drawbacks of DGM on clinical specimens as a diagnostic tool has been that both false positive and false negative diagnosis can be easily made even in experienced hands. ${ }^{[6,7]}$

Staining methods have been applied to increase the sensitivity of direct microscopic examination. These include immunofluorescence staining of bovine urine, water, and soil and immunoperoxidase staining of blood and urine. A variety of histopathological stains have been applied to the detection of leptospires in tissues. Leptospires were first visualized by silver staining, and the Warthin-Starry stain is widely used for histologic examination. Recently, immunohistochemical methods have been applied.

\section{Culture}

\section{Isolation of Leptospires}

Leptospiremia occurs during the first stage of the disease, beginning before the onset of symptoms, and ends by the first week of the illness. Therefore blood cultures should be taken as soon as possible after the patient's presentation. One or two drops of blood are inoculated into $10 \mathrm{ml}$ of semisolid medium containing 5-fluorouracil at the patient's bedside. For the greatest recovery rate, multiple cultures should be performed, but this is rarely possible. Inoculation of media with dilutions of blood samples may increase recovery. Rapid detection of leptospires by radiometric methods has been described. Leptospires survive in conventional blood culture media for a number of days. Rarely, leptospires have been isolated from blood weeks after the onset of symptoms. Isolation of leptospires from clinical samples gives a definitive diagnosis and also aids in identifying the prevalent serovar. ${ }^{[7]}$

Apart from blood, CSF and dialysate fluid can also be cultured during the first week of illness. Urine can be cultured from the beginning of the second week of symptomatic illness. The duration of urinary excretion varies but may last for several weeks. Survival of leptospires in voided human urine is limited, so urine should be processed immediately by centrifugation, followed by resuspending the sediment in phosphatebuffered saline (to neutralize the $\mathrm{pH}$ ) and inoculating into semisolid medium containing 5-fluorouracil.

Cultures are incubated at 28 to $30^{\circ} \mathrm{C}$ and examined weekly by dark-field microscopy for up to 13 weeks before being discarded. Contaminated cultures may be passed through a 0.2 $\mu \mathrm{m}$ or $0.45-\mu \mathrm{m}$ filter before subculture into fresh medium.

Though the use of culture confirms diagnosis, it is rarely used, as it is very tedious, complicated, expensive, technically demanding, time consuming, requiring prolonged incubation (minimum 1 month before declaring a sample negative) and may not be successful (low sensitivity). The organism also has a relatively long doubling time (6 to $8 \mathrm{~h}$ or more).Additionally they are highly infectious organisms requiring 'Biosafety level II' facilities.

\section{Identification of Leptospiral isolates}

Isolated leptospires are identified either by serological methods or by recently developed molecular techniques. Very few centres can perform these identification methods. The use of panels of monoclonal antibodies allows laboratories, which can perform the microscopic agglutination test to identify isolates with relative rapidity. Molecular methods have become more widely used and are discussed later in the review.

\section{Susceptibility testing}

Leptospires are susceptible to beta-lactams, macrolides, tetracyclines, fluoroquinolones and streptomycin. Problems in the determination of susceptibility include the long incubation time required, the use of media containing serum and the difficulty in quantifying growth accurately. These constraints have limited the development of rapid, standardized methods for susceptibility testing.

\section{Serology}

\section{Antigen detection}

Detection of leptospiral antigens in clinical material offer 
greater specificity than DGM while having the potential for greater sensitivity. Radioimmunoassay (RIA) can detect $10^{4}$ to $10^{5}$ leptospires/ml and an enzyme-linked immunosorbent assay (ELISA) method can detect $10^{5}$ leptospires/ml. A chemiluminescent immunoassay (CLIA) has been applied to human blood and urine but has been less sensitive than earlier ELISA. Recently, immunomagnetic antigen capture has been combined with fluoroimmunoassay to detect as few as $10^{2}$ leptospires/ml in urine of cattle infected with serovar hardjo.

\section{Antibody detection}

Most cases of leptospirosis are diagnosed by serology. ${ }^{[8,9,10]}$ Antibodies can become detectable by the 6th to 10th day of disease and generally reach peak levels within 3 to 4 weeks. Antibody levels then gradually recede but may remain detectable for years. Serological methods can be divided into two groups: those, which are genus specific and those which are serogroup specific. The definitive serological investigation in leptospirosis remains the microscopic agglutination test (MAT). ${ }^{[8,11,12,13]}$

\section{Microscopic agglutination test (MAT)}

The reference method for serological diagnosis of leptospirosis is the MAT, in which patient sera are reacted with live antigen suspensions of leptospiral serovars. After incubation, the serum-antigen mixtures are examined microscopically for agglutination and the titers are determined. Formerly, the method was known as the agglutination-lysis test because of the formation of lysis balls or lysis globules of cellular debris in the presence of high-titered antiserum. However, these are tightly agglutinated clumps of leptospires containing live cells and not debris. Several modifications of earlier methods led to a MAT method, which can be performed and read in microtiter trays. The MAT is read by dark-field microscopy. The end point is the highest dilution of serum at which $50 \%$ agglutination occurs. Because of the difficulty in detecting when $50 \%$ of the leptospires are agglutinated, the end point is determined by the presence of approximately $50 \%$ free, unagglutinated leptospires compared to the control suspension Considerable effort is required to reduce the subjective effect of observer variation, even within laboratories. Different laboratories use different cut-off titres ranging from 1 in 100 to 1 in 800 for diagnosis and may result in overdiagnosis and overestimation of disease burden. The importance of determination of base line titres in the community hence cannot be overemphasised. Interpretation of this test is complicated by the high degree of cross-reaction that occurs between different serogroups, especially in acute-phase samples. This is to some extent predictable, and patients often have similar titers to all serovars of an individual serogroup. Of note, "paradoxical" reactions in which the highest titers are detected to a serogroup unrelated to the infecting one, are also common The broad cross-reactivity in the acute phase, followed by relative serogroup specificity in convalescent-phase samples, results from the detection in the MAT of both IgM and IgG antibodies and the presence of several common antigens among leptospires.

Thus MAT is a complex test to control, perform, and interpret. Live cultures of all serovars required for use as antigens need to be maintained. This applies equally whether the test is performed with live or formalin-killed antigens.

Additionally, other drawbacks of MAT include the continuous risk of cross-contamination of the antigen cultures, necessitating periodic verification of each serovar. MAT titers are affected by the culture medium in which the antigens are grown. Moreover, the repeated weekly subculture of large numbers of strains presents hazards for laboratory workers.

\section{Other Serological Tests}

Because of the complexity of the MAT, rapid screening tests for leptospiral antibodies in acute infection have been developed. Complement fixation (CF) was widely used but methods were not standardized. CF tests have generally been replaced by ELISA methods.

IgM antibodies become detectable during the first week of illness allowing the diagnosis to be confirmed and treatment initiated while it is likely to be most effective. Antibody levels are generally low or absent during very early infection. IgM detection has repeatedly been shown to be more sensitive than MAT when the first specimen is taken early in the acute phase of the illness. However most of the commercially available ELISA kits use non-pathogenic L.biflexa patoc 1 strain as an antigen. The drawback of this test is that the infective serovar cannot be assessed. Though the test is more sensitive than MAT it is less specific.

An IgM-specific dot-ELISA has been developed in which polyvalent leptospiral antigen was dotted onto nitrocellulose filter disks in microtiter tray wells, allowing the use of smaller volumes of reagents. Further modifications of this approach have been used to detect $\operatorname{IgG}$ and $\operatorname{IgA}$, in addition to $\operatorname{IgM}$ and have employed an immunodominant antigen and a polyester fabric-resin support in place of nitrocellulose.

A commercial slide agglutination assay has been recently found to be as sensitive and specific as an IgM-ELISA, while remaining reactive for a shorter time after recovery than either the IgM-ELISA, or the MAT. ${ }^{[13]}$

A number of methods using sensitized red blood cells have been described. The extraction of an erythrocyte-sensitizing substance led to the development of both a hemolytic assay requiring complement and a hemagglutination assay, and a number of modifications of the latter have been reported. These assays detect both IgM and IgG antibodies. The number of antibody positive subjects in a population depends on two factors: disease prevalence and clinical criteria used to select the tested population.

The indirect hemagglutination assay (IHA) developed at CDC was shown to have a sensitivity of $92 \%$ and specificity of $95 \%$ compared with the MAT.

A microcapsule agglutination test using a synthetic polymer in place of red blood cells has been evaluated extensively. The 
microcapsule agglutination test is reportedly more sensitive than either the MAT or an IgM-ELISA in early acute phase samples, but failed to detect infections caused by some serovars. Advantages of this direct agglutination method is that it can be applied without modification to sera from other animal species.

Other techniques applied to the detection of leptospiral antibodies include immunofluorescence, RIA, counterimmunoelectrophoresis, and thin-layer immunoassay. These methods are not widely used.

\section{Molecular Diagnosis}

Leptospiral DNA has been detected in clinical material by dotblotting and in-situ hybridization. The sensitivity of ${ }^{32} \mathrm{P}-l$ labeled probes was approximately $10^{3}$ leptospires/ml, much lower than the sensitivity of PCR, and probes have not been used extensively for diagnosis since PCR became available.

Several primer pairs for PCR detection of leptospires have been described. Few of these have been shown to amplify leptospiral DNA from either human or veterinary clinical material, and of these, two methods have been subjected to extensive clinical evaluation. Both methods were found to be more sensitive than culture, but differences in analysis of the data render direct comparisons between the two approaches impossible.

A limitation of PCR-based diagnosis of leptospirosis is the inability of most PCR assays to identify the infecting serovar. While this is not significant for individual patient management, the identity of the serovar has significant epidemiological and public health value. Strategies designed to overcome this obstacle have included restriction endonuclease digestion of PCR products, direct sequencing of amplicons, and singlestrand conformation analysis (SSCP). Leptospiral genomospecies can be differentiated following PCR by electrophoresis in nondenaturing polyacrylamide gels, followed by silver staining, without the additional step of purification and denaturing.

PCR has also been used to distinguish pathogenic from nonpathogenic serovars. A fluorescent-probe 5' exonuclease PCR assay has also been described for the rapid detection of pathogenic leptospires.

\section{Molecular typing}

There has been great interest in molecular methods for identification and subtyping because of the difficulties associated with serological identification of leptospiral isolates,. Methods employed have included digestion of chromosomal DNA by restriction endonucleases (REA), restriction fragment length polymorphism (RFLP), ribotyping, pulsed field gel electrophoresis, and a number of PCR-based approaches.

A limiting factor in all methods which analyze chromosomal DNA is the requirement for large quantities of purified DNA. As a result, several methods based on the analysis of PCR-am- plified sections of leptospiral DNA have been employed DNA fingerprinting using arbitrary primers has been studied extensively, using different primers and conditions. Direct comparison between the results of these studies is therefore impossible, but it is clear that reproducibility is difficult to achieve without absolute standardization of experimental procedure. Profiles are affected markedly by the primer used, the quantity and quality of the DNA template, and the electrophoresis conditions. The greatest value of arbitrary primer techniques lie in their ability to differentiate between isolates when the range of potential serovars is limited, allowing rapid identification of freshly isolated strains.

\section{Conclusion}

Dark ground microscopy and serology form the basis of diagnosis of leptospirosis in most instances, however, knowledge of the efficacy of these and other tests including molecular tests will not only help in correct requisition of tests but also increase yield, thereby facilitating early and correct initiation of therapy.To conclude, this article has reviewed the various diagnostic techniques available for diagnosis of Leptospirosis and the advantages and drawbacks of each technique. Important facts that need emphasis pertain to DGM and serology.

Though DGM is useful for the diagnosis of leptospirosis it cannot be used as the sole diagnostic tool. The drawbacks of DGM on clinical specimens have been emphasized by stating that both false positive and false negative diagnosis can be easily made even in experienced hands. Though the use of culture confirms diagnosis it is impractical as it is expensive, complicated, technically demanding,time consuming requiring prolonged incubation (minimum 1 month before declaring a sample negative) and may not be successful (low sensitivity). A list of serological tests other than MAT and ELISA is given. Most cases of leptospirosis are diagnosed by serology. The role of microscopic agglutination test (MAT) in diagnosis and seroepidemiological studies is highlighted. However the lack of standardisation of base line titers in the community influences test validity and may may result in overdiagnosis and overestimation of disease burden.

\section{References}

1. Tappero JW, Ashford DA, Perkins BA. Leptospira species (leptospirosis), p. In: Mandell GL, Bennett JE, Dolin R, editors. Principles and practice of infectious diseases. $5^{\text {th }}$ edn. Philadelphia:Churchill Livingstone: 2000 . p. 2495 501

2. Dutta TK, Christopher M. Leptospirosis - an overview. J Assoc Physic Ind 2005;53:545-51.

3. Clerke AM, Leuva AC, Joshi C, Trivedi SV. Clinical profile of leptospirosis in South gujarat. J Postgrad Med 2002;48:117-8.

4. Shah I, Warke S, Deshmukh CT, Kamat JR. Leptospirosis - an under-diagnosed clinical condition. J Postgrad Med 1999;45:93-4.

5. Levett PN. Leptospirosis. Clin Microbiol Rev 2001;14:296-326.

6. O'Keefe JS. A brief review on the laboratory diagnosis of leptospirosis. N Z Vet J 2002;50:9-13

7. Effler PV Bogard AK, Domen HY, Katz AR, Higa HY, Sasaki DM. Evaluation of Eight Rapid Screening Tests for Acute Leptospirosis in Hawaii. J Clin Microbiol 2002;40:1464-9

8. Angela P, Brandão AP, Camargo ED, da Silva ED, Marcos V, Silva MV, et al. Macroscopic Agglutination Test for Rapid Diagnosis of Human Leptospirosis. J Clin Microbiol 1998;36:3138-42.

9. Effler PV, Domen HY, Bragg SL, Aye T, Sasaki DM. Evaluation of the Indirect Hemagglutination Assay for Diagnosis of Acute Leptospirosis in Hawaii. J Clin Microbiol 2000;38:1081-4. 
10. Flannery B, Costa D, Carvalho FP, Guerreiro H, Matsunaga J, Da Silva ED, et al. Evaluation of Recombinant Leptospira Antigen-Based Enzyme-Linked Immunosorbent Assays for the Serodiagnosis of Leptospirosis. J Clin Microbiol 2001;39:3303-10.

11. Arimitsu $Y$, Kmety E, Ananyina Y, Baranton G, Ferguson IR, Smythe L, et al. Evaluation of the one-point microcapsule agglutination test for diagnosis of leptospirosis 1994;72:395-9.
12. Brandão AP, Camargo ED, da Silva ED, Silva MV, Abrão RV. Macroscopic agglutination test for rapid diagnosis of human leptospirosis. J Clin Microbiol 1998; $36: 3138-42$

13. Cumberland PC, Everard CO, Levett PN. Assessment of the efficacy of the IgM enzyme-linked immunosorbent assay (ELISA) and microscopic agglutination test (MAT) in the diagnosis of acute leptospirosis. Am J Trop Med Hyg 1999;61:731-4. 